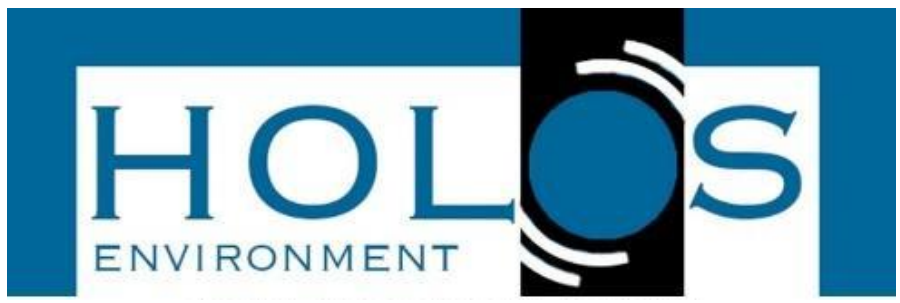

\title{
ANÁLISE TEMPORAL DA TEMPERATURA DE SUPERFÍCIE OBTIDA POR SENSORIAMENTO REMOTO
}

\section{TEMPORAL ANALYSIS OF SURFACE TEMPERATURE OBTAINED BY REMOTE SENSING}

\author{
Darllan Collins da Cunha e Silva ${ }^{1}$; Renan Angrizani de Oliveira²; Erik de Lima \\ Andrade $^{2}$; Érico Tadao Teramoto ${ }^{1}$; Vanessa Cezar Simonetti
}

Artigo recebido em: 31/01/2020 e aceito para publicação em: 19/02/2020.

DOI: http://dx.doi.org/10.14295/holos.v20i2.12374

Resumo: É crescente a preocupação com o aumento da temperatura que vem ocasionando diversos impactos à fauna e flora, neste sentindo, dados de sensoriamento remoto (SR) tem sido amplamente utilizados para a aquisição de informações sobre a temperatura da superfície terrestre. Portanto, o estudo objetivou correlacionar os dados de temperatura de uma estação automática da Companhia Ambiental do Estado de São Paulo (Cetesb), localizada no município de Sorocaba com os dados obtidos remotamente por meio do processamento digital de imagens do satélite Landsat-8 OLI entre os anos de 2013 a 2017. Para o processamento das imagens foi utilizado o software ArcGIS 10.4.1, utilizando os parâmetros fixos de conversão de níveis de cinza da imagem para radiância e posterior conversão dos dados em valores de temperatura. Para verificar a variação dos dados obtidos por SR comparados a estação de monitoramento, foi verificada a Raiz do Erro Quadrático Médio (REQM), coeficiente de correlação Pearson ( $r$ e o índice de concordância (IC). Os valores obtidos de REQM indicam uma variação média de temperatura de $2,74 \stackrel{\circ}{\circ}$, corroborando com os valores de $\mathrm{r}^{2}$ igual a 0,664 que indicam que $66,4 \%$ dos valores obtidos para a temperatura remotamente são diretamente relacionados aos valores obtidos na estação de monitoramento da Cetesb, apresentando valores de IC com concordância positiva de 0,88 . Portanto, os resultados encontrados se mostraram satisfatórios para uma melhor aquisição de valores de temperatura obtidos remotamente indicando boa correlação com os dados obtidos pela estação de monitoramento da Cetesb.

Palavras-chave: Sensoriamento remoto. Infravermelho térmico. Correção atmosférica. Erro Quadrático.

\begin{abstract}
There is a growing concern with respect to the temperature increases that has caused several impacts on fauna and flora. In this sense, remote sensing (SR) data has been widely used for the acquisition of information on the Earth's surface temperature. Thus, this study aimed to correlate the temperature data of an automatic station of the Companhia Ambiental do Estado de São Paulo (Cetesb), located in Sorocaba, with data obtained remotely through digital image processing of the Landsat 8 satellite between the years 2013 to 2017. ArcGIS 10.4.1 software was used for image processing, using the fixed parameters of conversion of gray image levels to radiance and subsequent conversion of data into temperature values. To verify the variation of the data obtained by RS compared to the monitoring station, the Root Mean Square Error (RMSE), Pearson correlation coefficient ( $r$ ) and index of agreement (d-index) were verified. The obtained RMSE values indicate an average temperature variation of $2.74^{\circ} \mathrm{C}$, corroborating the $\mathrm{r}^{2}$ values of 0.664 , which indicates that

1 Universidade Estadual Paulista "Júlio de Mesquita Filho" (UNESP) - Campus de Registro, SP. E-mails: (darllan.collins@unesp.br, erico.teramoto@unesp.br)

2 Universidade Estadual Paulista "Júlio de Mesquita Filho" (UNESP) - Campus de Sorocaba, SP. E-mails: (renan angrizani@hotmail.com, eng.erik@hotmail.com, va simonetti@hotmail.com)
\end{abstract}


$66.4 \%$ of the values obtained for the temperature remotely are directly related to the values obtained at the Cetesb monitoring, presenting positive agreement $d$-index values of 0.88 . Therefore, the results were satisfactory for a better acquisition of temperature values obtained remotely indicating a good correlation with the data obtained by the Cetesb monitoring station.

Keywords: Remote sensing. Thermal infrared. Atmospheric correction. Square Error.

\section{INTRODUÇÃO}

O aumento das temperaturas se tornou um dos principais problemas ambientais da atualidade ocasionando diversos impactos à fauna e flora, e mesmo um pequeno aumento de temperatura das condições climáticas locais pode resultar na perda de biodiversidade e acarretar diversos prejuízos econômicos e à saúde da população (AHMED et al., 2015; ALBUQUERQUE; LOPES, 2016; ARNELL et al., 2019).

As ferramentas de sensoriamento remoto vêm sido amplamente utilizadas para a aquisição dos dados de temperatura da superfície, pois possibilitam o monitoramento da temperatura de forma remota e com um menor custo de operação (LIU, 2015). Estudos como os de Coelho e Correa (2013) e Corrêa e Corrêa (2012) destacam a utilização de sensoriamento remoto para obtenção de dados de temperatura da superfície através de imagens de satélite para identificação de áreas de risco, ilhas de calor, cobertura do solo e diversos projetos de caráter ambiental.

Neste sentido, estudos recentes têm mostrado a aplicação da temperatura de superfície (TS) para diversas aplicações. Filgueiras et al. (2019) realizaram a simulação de erros em temperaturas de superfície para verificação das consequências da atual evapotranspiração e seus possíveis efeitos utilizando o modelo SAFER (Simple Algorithm for Evapotranspiration Retrieving). Enquanto Nguyen et al. (2019) utilizaram a técnica de sensoriamento remoto para monitorar as alterações de temperatura da superfície da terra na estação de seca na região sudeste do Vietnã, entre o período de 2015 a 2016. Já os estudos realizados por Silvestri et al. (2019) utilizaram a análise térmica obtida por sensoriamento remoto para testar a capacidade em múltiplas escalas e multitemporal no monitoramento vulcânico da llha Vulcano na Itália.

Diante do exposto, o presente estudo possui o objetivo de realizar o levantamento dos dados de temperatura de uma estação automática da Companhia Ambiental do Estado de São Paulo (Cetesb), localizada no município de Sorocaba e correlacioná-los com os 
dados obtidos de forma remota pelo processamento digital de imagem de satélite com o auxílio de um SIG entre os anos de 2013 a 2017.

\section{MATERIAL E MÉTODOS}

\section{1 Área de Estudo}

A estação da rede automática de monitoramento da Companhia Ambiental do Estado de São Paulo (Cetesb) localizada na Região Metropolitana de Sorocaba (RMS) no município de Sorocaba (SP) situada na rua Nhonhô Pires, 260, Vila Lucy, nas coordenadas UTM, 2468630 e 7398684S, Fuso 23S (Figura 1).

Figura 1 - Localização da estação pertencente a rede automática de monitoramento da Cetesb

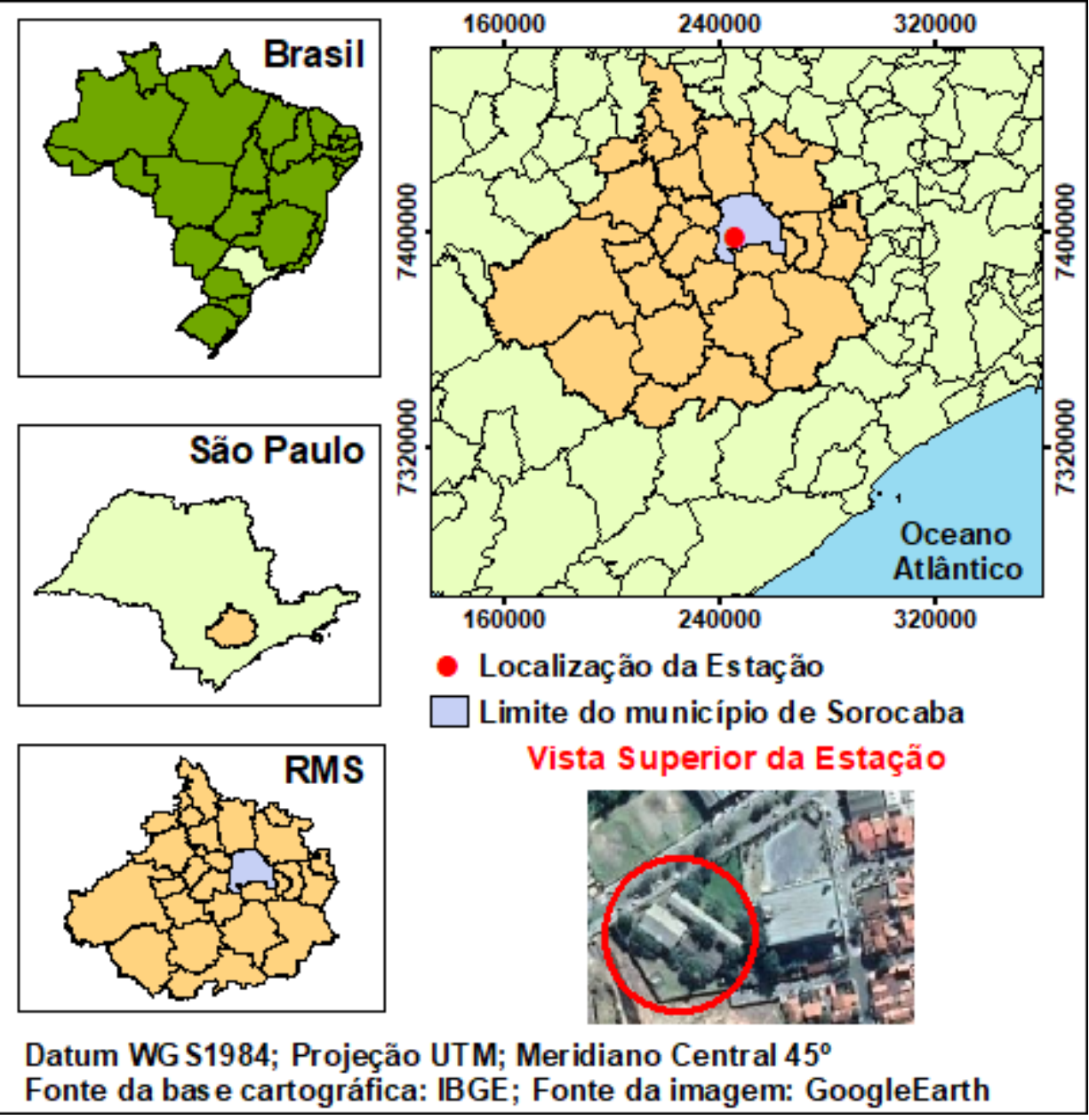

Fonte: Elaborado pelos autores 
O clima do município de Sorocaba, segundo Köeppen-Geiger, é dominante do tipo "Cfa", caracterizado como subtropical quente, inverno não muito seco, com uma precipitação média anual de $1.400 \mathrm{~mm}$, possuindo, também, uma faixa mais ao norte com características de clima tipo "Cwa", caracterizado como clima temperado chuvoso e quente, com uma pluviometria inferior a $30 \mathrm{~mm}$ no mês predominantemente mais seco (SIMONETTI et al., 2018).

\subsection{Procedimento Metodológico}

A primeira etapa consistiu na aquisição de informações de temperatura no período de 01 de janeiro de 2013 a 31 de dezembro de 2017 da estação da rede automática (CETESB, 2018). Em seguida foram realizadas as aquisições das imagens orbitais digitais da cidade de Sorocaba, do mesmo período de 2013 a 2017, através do site do Serviço Geológico dos Estados Unidos (USGS, 2018), referentes ao satélite Landsat-8 OLI na banda 10, faixa do infravermelho termal (10.6 - $11.19 \mu \mathrm{m}$ ) do sensor TIRS (Thermal Infrared Sensor), nas órbitas 219 e 220 do ponto 76, com resolução espacial de 100 m. A revisita do satélite ocorre em um intervalo de 16 dias e passa pelo local de estudo próximo das $13 \mathrm{~h}$ no horário Greenwich Mean Time (GMT) e às 10h do horário de Brasília.

Após a aquisição das imagens, foram adotados alguns critérios de seleção das quais seriam efetivamente utilizadas no estudo. O primeiro critério adotado foi a quantidade de nuvens presentes nas imagens de satélite, as que possuíam valores acima de $50 \%$ de cobertura de nuvem foram descartadas. Também foram excluídas as imagens que apresentaram nuvens na mesma coordenada do ponto analisado, independentemente de possuírem valores inferiores a $50 \%$ de cobertura de nuvem.

Como a banda 10 pondera valores da temperatura de superfície dos alvos sobre a superfície terrestre, faz-se necessário uma correção para obter o valor real da temperatura, visto que esta banda avalia a emissão do calor (Chander et al., 2009).

Para o tratamento do dado raster obtido das imagens do Landsat 8 foi utilizado o software ArcGIS 10.4.1. Para isso, utilizou-se os parâmetros fixos de conversão de níveis de cinza da imagem, para radiância. Esse procedimento pode ser observado através da Equação (1), disponibilizada através do Serviço Geológico Americano (USGS).

$\mathrm{L} \lambda=\mathrm{ML}{ }^{*} \mathrm{Qcal}+\mathrm{AL}$ 
Sendo:

L $\lambda$ expressa o valor da radiância espectral do sensor de abertura (W/m².sr. $\mu m)$

ML é o fator multiplicativo de redimensionamento de banda 10 (3,3420E-04)

Qcal é o valor quantizado calibrado pelo pixel em DN (banda 10)

$A L$ é o fator de redimensionamento aditivo específico da banda $10(0,10)$

Posteriormente, a radiância espectral foi convertida em temperatura conforme demonstrado na Equação (2).

$$
\mathrm{T}=\frac{\mathrm{K} 2}{\ln \left(\frac{\mathrm{K} 1}{\mathrm{~L} \lambda}+1\right)}
$$

Sendo:

T é o valore de temperatura efetiva no satélite em Kelvin (K);

K1 é a constante de calibração 1 (774,89 K);

K2 é a constante de calibração 2 (1321,08 K);

$\mathrm{L} \lambda$ expressa o valor da radiância espectral $\left(\mathrm{W} / \mathrm{m}^{2} . \mathrm{Sr} . \mu \mathrm{m}\right)$.

Os valores de temperatura são obtidos em Kelvin, sendo necessário convertê-los para Celsius $\left({ }^{\circ} \mathrm{C}\right)$, que é a mesma unidade de medida de temperatura da estação da rede automática da Cetesb.

Após a obtenção dos valores de temperatura do Landsat 8 foi realizada uma comparação com os dados obtidos pela estação da Cetesb. Para esta comparação utilizouse a equação da Raiz do Erro Quadrático Médio (REQM), do inglês Root Mean Square Error (RMSE), sendo expresso pela Equação 3.

$R E Q M=\left[\frac{1}{n} \sum_{\mathrm{i}=1}^{\mathrm{n}}\left(E_{i}-O_{i}\right)^{2}\right]^{\frac{1}{2}}$

Sendo:

REQM é a Raiz do Erro Quadrático Médio das temperaturas;

$E_{i}$ é o valor obtido pelo sensor TIRS do satélite Landsat 8;

Oi é o valor obtido pela estação automática de monitoramento da Cetesb;

n expressa o número de observações. 
Segundo Xu et al. (2016) e Silva et al. (2018) quanto menor o valor de REQM, menor a variabilidade indicada entre os valores estimados e os obtidos.

Também foi calculado o índice de concordância (IC), conforme Equação 4, que é uma medida do grau em que os dados estimados estão ou não livres de certos erros. Esse índice varia de 0 a 1 , sendo que para os valores iguais a 1 indica-se uma perfeita concordância entre os valores observados e os estimados, e para valores iguais a 0 uma total discordância entre os valores (WILLMOTT, 1985).

$$
I C=1-\frac{\sum_{\mathrm{i}=1}^{\mathrm{n}}\left(E_{i}-O_{i}\right)^{2}}{\sum_{\mathrm{i}=1}^{\mathrm{n}}\left(\left|E_{i}-\bar{O}\right|+\left|O_{i}-\bar{O}\right|\right)^{2}}
$$

Sendo:

IC é o índice de concordância;

$E_{i}$ é o valor obtido pelo sensor TIRS do satélite Landsat 8;

Oi é o valor pela estação automática de monitoramento da Cetesb;

n é o número de observações;

O é a média aritmética dos valores observados pela estação da Cetesb.

Para análise do grau de correlação entre os dados medidos pela estação da Cetesb e por sensoriamento remoto, foi calculado o coeficiente de correlação de Pearson ( $r$ ), que mede o grau da correlação entre duas variáveis. O coeficiente varia de -1 a 1, em que para valores iguais a 0 há uma ausência de correlação, -1 apresenta uma correlação negativa perfeita e 1 apresenta uma correlação positiva perfeita (SILVA et al., 2014). Para avaliar a significância do teste foi utilizada a distribuição de probabilidade de $F$ para um nível de decisão de $\alpha$ igual 0,05 .

\section{RESULTADOS E DISCUSSÃO}

A partir da análise dos dados do satélite Landsat 8, foram obtidas no total 214 imagens entre o período de 2013 a 2017, sendo que conforme os critérios de seleção adotados, foram consideradas apenas 94 imagens (44\%) no estudo.

De modo geral, houve um equilíbrio entre a quantidade de imagens usadas e perdidas durante o período avaliado anualmente, exceto para o ano de 2014, conforme ilustrado na Figura 2. 
Figura 2 - Comparativo anual da quantidade de imagens utilizadas do satélite Landsat 8

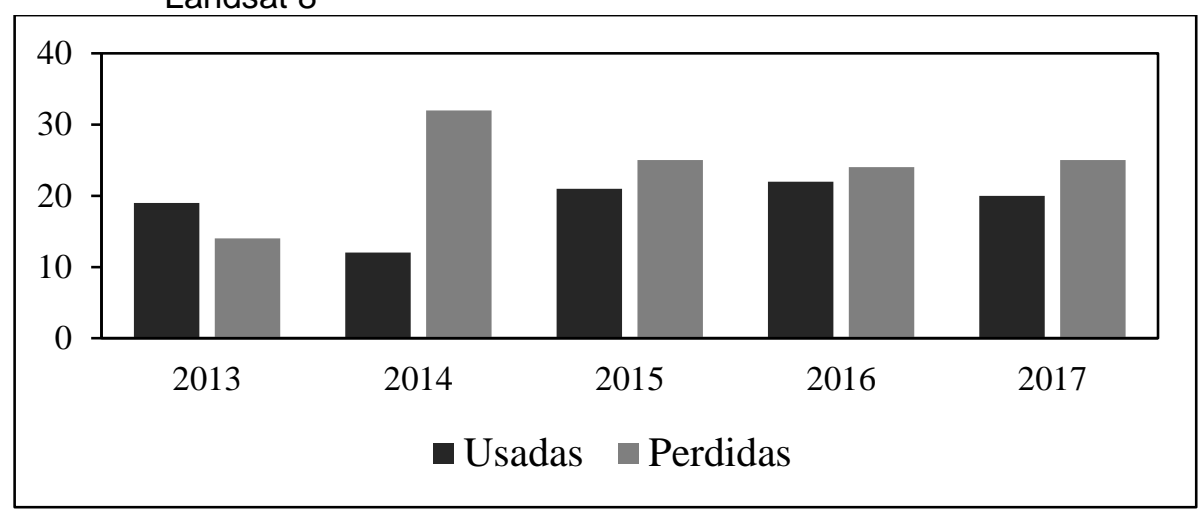

Fonte: Elaborado pelos autores

A partir dos critérios de seleção das imagens foi verificado que o ano de 2014, do total de imagens analisadas neste período, $72,7 \%$ das imagens foram desconsideradas, 0 que representa um valor superior em relação aos demais períodos analisados. As perdas das imagens estão relacionadas às chuvas e a presença de nuvens que afetaram a medição realizada pelo satélite Landsat 8 , conforme demonstrado Figura 3 , que representa a precipitação média entre os anos de 2013 a 2017.

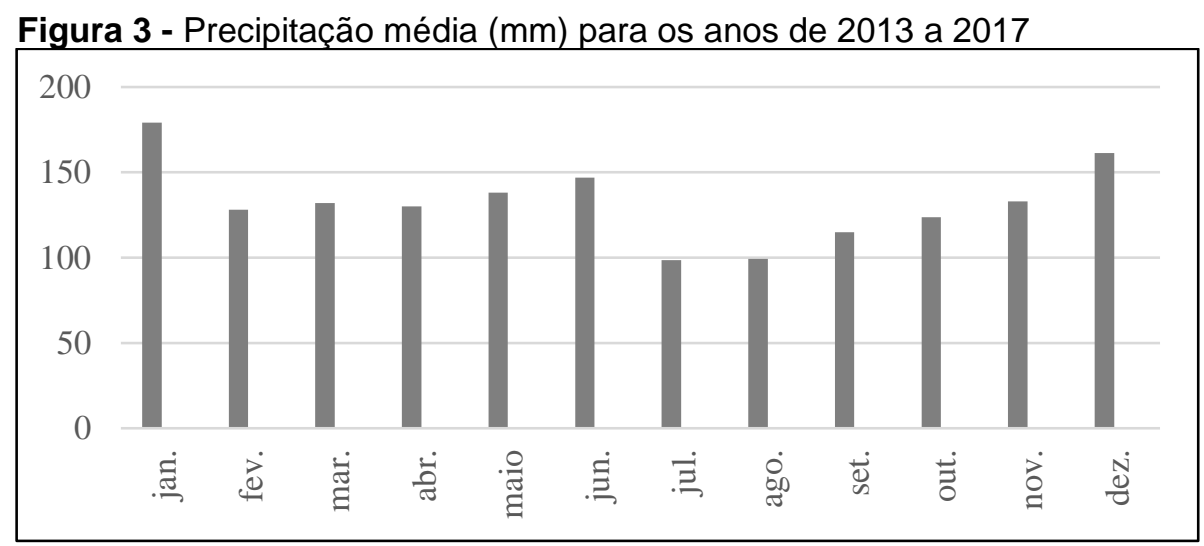

Fonte: Elaborado pelos autores

A nuvem e sua sombra no terreno causam obstruções de feições nas imagens, assim como a umidade, velocidade do vento e precipitação, sendo este último parâmetro verificado no Instituto Nacional de Meteorologia (INMET, 2018). A interferência na análise quantitativa ocorre quando os pixels que são interferidos por nuvem e sombra são tratados como válidos mesmo que não representem a imagem de forma correta.

Os períodos mais afetados avaliados foram os meses de janeiro, março, novembro e dezembro, correspondentes as estações de primavera e verão no Hemisfério Sul, que 
possuem maior incidência de chuvas no município de Sorocaba. Juntos estes quatro meses destacados, representam um total de 50 imagens perdidas, equivalentes a $42 \%$ do total de imagens perdidas, corroborando com as informações das condições climáticas de Sorocaba apresentados para os anos de 2013 a 2017, conforme pode ser observado na Figura 3.

Após a seleção das imagens foi verificada a variabilidade entre os valores estimados e obtidos por meio da Raiz do Erro Quadrático Médio (REQM). O valor obtido para o REQM corresponde a variação média de temperatura de $2,74 \stackrel{\circ}{\circ} \mathrm{C}$ para os valores obtidos da estação de monitoramento da Cetesb.

Para verificar se há uma correlação entre as temperaturas obtidas pela estação da Cetesb e por sensoriamento remoto, foi aplicado um teste de correlação Pearson ( $r$ ) para o valor médio dos dados, no período de 2013 a 2017.

Por meio da correlação entre os valores da temperatura captadas pela estação da Cetesb e os valores obtidos pelas imagens de satélite do Landsat 8 apresentadas na Figura 4, foi possível verificar que a correlação é positiva e que a correlação é significativa, uma vez que o valor do teste de probabilidade de $F$ obtido foi de 179,50 para um $p$ inferior a 0,0001 . Neste texto foi suprimido um outlier, onde a temperatura medida pela estação foi de $13,6^{\circ} \mathrm{C}$ e a temperatura estimada pela imagem de satélite foi de $21,1^{\circ} \mathrm{C}$. Este erro pode estar associado a resolução da banda $10(100 \mathrm{~m})$ devido ao tamanho do pixel, pois a área monitorada é urbana e fatores externos a estação como a temperatura do asfalto, por exemplo, podem elevar a temperatura neste pixel.

Figura 4 - Correlação dos valores de temperatura

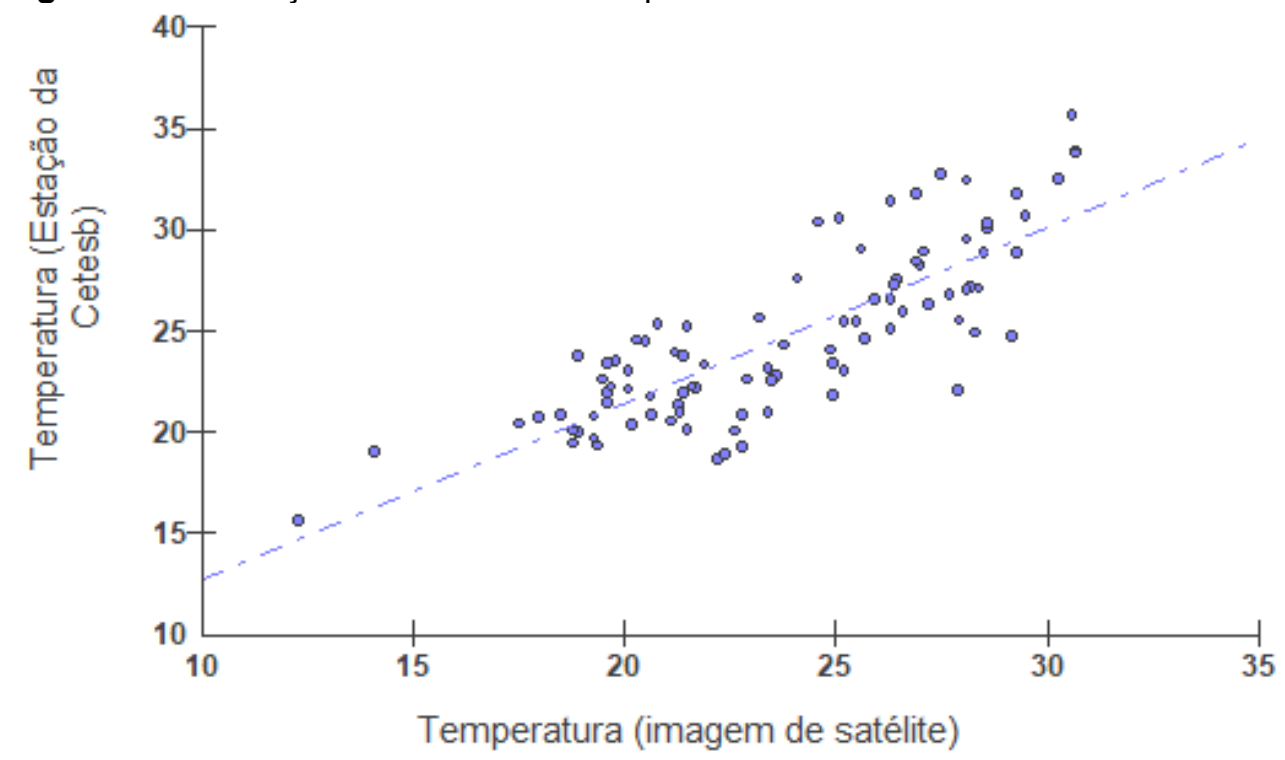

Fonte: Elaborado pelos autores 
O coeficiente de correlação Pearson ( $r$ ) encontrado foi de 0,815 , enquanto que, 0 coeficiente de determinação $\left(r^{2}\right)$ foi de 0,664 , ou seja, $66,4 \%$ dos valores obtidos para a temperatura por meio de imagens de satélite são diretamente relacionados aos valores captados pela estação, entretanto, $19,8 \%$ dos valores destoam do valor captado pela estação, podendo ser explicados pela resolução espacial da imagem que é de $100 \mathrm{~m}$ com reamostragem de $30 \mathrm{~m}$, que se refere a uma área de $900 \mathrm{~m}^{2}$, no qual o valor da temperatura é médio para área e no caso da estação é pontual. Além disso, há a influência de aerossóis presentes na atmosfera que interferem no balanço energético.

Estudos como de Cherif et al. (2019), que correlacionaram a temperatura da água do rio Boukhalef, obtida através de sensoriamento remoto, com a concentração de bactérias medidas em in situ, obteve um valor de coeficiente de correlação de 0,85 , muito próximo do encontrado neste estudo.

Outro valor semelhante foi observado nos estudos de Hofierka et al. (2020), onde os autores compararam valores modelados da temperatura terrestre com os derivados do Landsat 8, e obtiveram o valor de 0,88 de correlação. Vanhellemont (2020) avaliando o desempenho do sensor infravermelho termal (TIRS) a bordo do Landsat 8 para recuperação da temperatura de superfície terrestre e da água, chegou a valores acima de 0,94 de coeficiente de correlação nas configurações aplicadas e erro quadrático médio de $0,7 \mathrm{Ke}$ $1 \mathrm{~K}$ para as bandas 10 e 11.

O índice de concordância calculado neste estudo para os dados da estação da Cetesb e obtidos por sensoriamento remoto apresentaram um valor positivo de 0,88 , valor superior ao encontrado por Camparotto et al. (2013) que compararam dados de temperatura de superfície com dados de temperatura do satélite Aqua para o sensor Modis. Rampazo et al. (2019) compararam dados de superfície de 31 estações meteorológicas no estado de São Paulo com dados modelados e de satélite, chegando a índices de concordância de 0,63 e 0,82 para dados diários e decendiais respectivamente, valores inferiores ao encontrado neste estudo.

Portanto, o presente estudo possibilitou uma melhor estimativa de temperatura obtida por sensoriamento remoto para a região estudada por meio da Raiz do Erro Quadrático Médio (REQM), sendo verificada uma boa concordância entre os valores observados e os estimados conforme verificado pelo índice de concordância. Desta forma, a metodologia apresentada se mostra viável para a aplicação em outros intervalos de tempo e locais, reduzindo também o custo de aquisição dos dados de temperatura, possibilitando 
uma maior área de abrangência de dados, contribuindo dessa forma para novos estudos e auxiliando na tomada de decisão.

\section{CONCLUSÕES}

A Raiz do Erro Quadrático Médio (REQM) obtida para este estudo, apresentou uma variação média de temperatura de $2,74^{\circ} \mathrm{C}$, corroborando com os valores encontrados para o coeficiente de determinação $\left(\mathrm{r}^{2}\right)$ de 0,664 , ou seja, $66,4 \%$ dos valores obtidos para a temperatura por meio de imagens de satélite são diretamente relacionados aos valores captados pela estação de monitoramento da Cetesb, apresentando uma correlação positiva e significativa, uma vez que o valor do teste de probabilidade de $F$ obtido foi de 179,5 , corroborando com os valores do índice de concordância calculados que apresentam uma concordância positiva de 0,88 . Portanto, os resultados encontrados para o período de 2013 a 2017 se mostraram satisfatórios para uma melhor aquisição de valores de temperatura obtidos remotamente por meio das imagens do satélite do Landsat 8, apresentando uma boa correlação com os dados obtidos pela estação de monitoramento da Cetesb localizada em Sorocaba, mesmo com a perda de $56 \%$ das imagens devido as condições climáticas, onde nas estações da primavera e verão devido a maior incidência de chuvas no município de Sorocaba houve um menor aproveitamento das imagens.

\section{REFERÊNCIAS}

AHMED, A. Q.; OSSEN, D. R.; JAMEI, E.; MANAF, N. A.; SAID, I.; AHMAD, M. H. Urban surface temperature behaviour and heat island effect in a tropical planned city. Theoretical and applied climatology, v. 119, n. 3-4, p. 493-514, 2015. https://doi.org/10.1007/s00704-014-1122-2

ALBUQUERQUE, M. M.; LOPES, W. G. R. Influência da vegetação em variáveis climáticas: estudo em bairros da cidade de Teresina, Piauí. Raega-O Espaço Geográfico em Análise, v. 36, p. 38-68, 2016. https://doi.org/10.5380/raega.v36i0.39719

ARNELL, N. W.; LOWE, J. A., CHALLINOR, A. J.; OSBORN, T. J. Global and regional impacts of climate change at different levels of global temperature increase. Climatic Change, v. 155, n. 3, p. 377-391, 2019.

CAMPAROTTO, L. B.; BLAIN, G. C.; GIAROLLA, A.; ADAMI, M.; CAMARGO, M. B. P. Validação de dados termopluviométricos obtidos via sensoriamento remoto para o Estado de São Paulo.

Revista Brasileira de Engenharia Agrícola e Ambiental, v. 17, n. 6, p. 665-671, 2013. 
CETESB, Companhia Ambiental do Estado de São Paulo. Qualidade do Ar (2018). Disponível em: https://cetesb.sp.gov.br/ar/qualar/. Acesso em: 19 fev. 2018.

CHANDER, G.; MARKHAM, B. L.; HELDER, D. L. Summary of current radiometric calibration coefficients for Landsat MSS, TM, ETM+, and EO-1 ALI sensors. Remote sensing of

environment, v. 113, n. 5, p. 893-903, 2009. https://doi.org/10.1016/i.rse.2009.01.007

CHERIF, E. K.; SALMOUN, F.; MESAS-CARRASCOSA, F. J. Determination of Bathing Water Quality Using Thermal Images Landsat 8 on the West Coast of Tangier: Preliminary Results. Remote Sensing, v. 11, n. 8, p. 972, 2019. https://doi.org/10.3390/rs11080972

COELHO, A. L. N.; CORREA, W. S. C. Temperatura de Superfície Celsius do Sensor TIRS/Landsat-8: metodologia e aplicações. Revista Geográfica Acadêmica, v. 7, n. 1, p. 31-45, 2013.

CORRÊA, P. B.; CORRÊA, J. A. J. Análise da temperatura de superfície da área urbana de Santarém através de imagens termais do Landsat 5. Revista Geonorte, v. 2, n. 4, p. 714-722, 2012.

FILGUEIRAS, R.; MANTOVANI, E. C.; ALTHOFF, D.; DIAS, S. H. B.; CUNHA, FERNANDO F. Sensitivity of evapotranspiration estimated by orbital images under influence of surface temperature. Engenharia Agrícola, v. 39, n. spe, p. 23-32, 2019. https://doi.org/10.1590/18094430-eng.agric.v39nep23-32/2019

HOFIERKA, J.; GALLAY, M.; ONAČILLOVÁ, K.; HOFIERKA JR, J. Physically-based land surface temperature modeling in urban areas using a 3-D city model and multispectral satellite data. Urban Climate, v. 31, p. 100566, 2020. https://doi.org/10.1016/j.uclim.2019.100566

INMET, Instituto Nacional de Meteorologia. Estações Automáticas. Disponível em: http://www.inmet.gov.br/portal/index.php?r=estacoes/estacoesAutomaticas. Acesso Out. 2018.

LIU, W. T. H. Aplicações de sensoriamento remoto. 2 ed. São Paulo: Oficina de Textos, 2015.

NGUYEN, Q. K.; TRINH L. H.; DAO K. H.; DANG N. D. Land surface temperature dynamics in dry season 2015-2016 according to Landsat 8 data in the South-East region of Vietnam. Geography, Environment, Sustainability, v. 12, n. 1, p. 75-87, 2019. https://doi.org/10.24057/2071-9388$\underline{2018-06}$

RAMPAZO, N. A. M., PICOLI, M. C. A., CAVALIERO, C. K. N. Comparação entre dados meteorológicos provenientes de sensoriamento remoto (modelados e de satélites) e de estações de superfície. Revista Brasileira de Geografia Física, v. 12, n. 02, p. 412-426, 2019. https://doi.org/10.26848/rbgf.v12.2.p412-426

SILVA, D. C. C.; ALBUQUERQUE FILHO, J. L.; OLIVEIRA, R. A.; LOURENÇO, R. W. Proposta metodológica para análise espacial de nutrientes do solo em bacias hidrográficas. Sociedade \& Natureza, v. 30, p. 85-107, 2018. https://doi.org/10.14393/SN-v30n3-2018-5

SILVA, D. C. C.; LOURENÇO, R. W.; DONALISIO, M. R.; CORDEIRO, R. Análise da relação entre a distribuição espacial das morbidades por obesidade e hipertensão arterial para o estado de São Paulo, Brasil, de 2000 a 2010. Ciência e Saúde Coletiva, v. 19, p. 1709-1719, 2014.

http://dx.doi.org/10.1590/1413-81232014196.15002013 
SILVESTRI, M.; RABUFFI, F.; PISCIOTTA, A.; MUSACCHIO, M.; DILIBERTO, I. S.; SPINETTI, C.; LOMBARDO, V.; COLINI, L.; BUONGIORNO, M. F. Analysis of thermal anomalies in volcanic areas using multiscale and multitemporal monitoring: vulcano island test case. Remote Sensing, v. 11, n. 2, p. 134, 2019. https://doi.org/10.3390/rs11020134

SIMONETTI, V. C.; SILVA, D. C. C.; OLIVEIRA, R. A.; SABONARO, D. Z.; ROSA, A. H. Análise da suscetibilidade do solo a processos erosivos do Parque Natural Municipal Corredores de Biodiversidade (PNMCBIO) de Sorocaba (SP). Raega-O Espaço Geográfico em Análise, v. 44, p. 169-180, 2018. https://doi.org/10.5380/raega.v44i0.48838

USGS, Geological Survey / Serviço de Levantamento Geológico Americano (2018). Disponível em: https://earthexplorer.usgs.gov/. Acesso em: 22 jun. 2018.

VANHELLEMONT, Q. Automated water surface temperature retrieval from Landsat 8/TIRS.

Remote Sensing of Environment, v. 237, p. 111518, 2020.

https://doi.org/10.1016/j.rse.2019.111518

WILLMOTT, C. J.; ACKLESON, S. G.; DAVIS, J. J.; FEDDEMA, K. M.; KLINK, D. R.; LEGATES, D. R.; O'DONNELL, J.; ROWE, C. M. Statistics for the evaluation and comparison of models. Journal of Geophysical Research, v. 90, n. C5, p.8995-9005, 1985. https://doi.org/10.1029/JC090iC05p08995

XU, W.; ZOU, Y.; ZHANG, G.; LINDERMAN, M. A comparison among spatial interpolation techniques for daily rainfall data in Sichuan Province, China. International Journal of Climatology, v. 35, n. 10, p. 2898-2907, 2015. https://doi.org/10.1002/joc.4180 